\title{
New algorithm successfully identifies infants at risk of progressive necrotising enterocolitis
}

The management of necrotising enterocolitis (NEC) could be advanced thanks to a new algorithm that combines clinical parameters with urine peptide biomarkers.

What triggers the onset and leads to progression of NEC remains unknown, making it difficult to identify which infants will need surgery and when it should be performed. "We were motivated to develop a vehicle that could allow clinicians to alter the course of the disease if the highest risk infants could be targeted," explains Karl Sylvester,

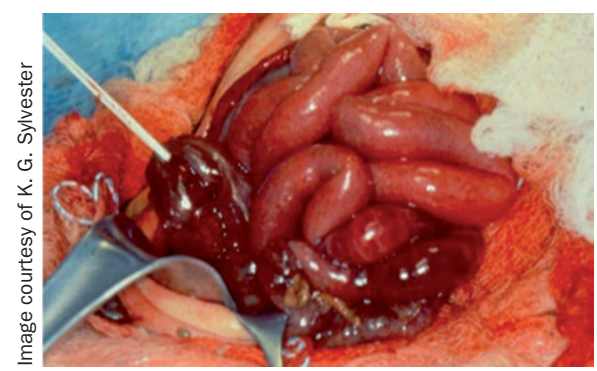

member of a consortium of academic children's hospitals set up to study current best practices for NEC. "A simple practical approach would include transfer to a higher level of care in those institutions that do not possess surgical capabilities for neonates."

The team constructed a multivariate predictor of progressive NEC using 27 clinical parameters. In addition, they analysed the urine peptidomes of infants with NEC by using a mass spectrometry based discovery platform. Three of the candidate urine peptides identified-FGA1826, FGA1883 and FGA2659, all fibrinogen peptidesdiscriminated accurately between medical NEC and surgical NEC. The final stage was to combine the clinical parameters and urine biomarkers in an ensemble algorithm.

The individual ROC curves for the clinical parameters and biomarkers had an AUC of 0.817 and 0.856 , respectively.
Using clinical parameters alone meant that $40.1 \%$ of infants were classified as being at 'indeterminate' risk of progressive NEC. By contrast, the ensemble model combining the urine peptide biomarkers and the clinical risk factors successfully separated all cases of medical NEC from surgical NEC.

Further work is now needed to validate and test the ensemble algorithm prospectively, and the possibility of incorporating other or different peptides also needs to be considered. Nonetheless, "for the first time, investigators and clinicians can think about alternative strategies for management of patients with NEC," concludes Sylvester.

\section{Natalie J. Wood}

Original article Sylvester, K. G. et al. A novel urine peptide biomarker-based algorithm for the prognosis of necrotising enterocolitis in human infants. Gut doi:10.1136/ gutjnl-2013-305130 